COLLOQUE DE PHYSIQUE

Colloque C2, supplément au $n^{\circ} 2$, Tome 51, Février 1990

ler Congrès Français d'Acoustique 1990

\title{
ÉTUDE ET RÉALISATION D'UN SYSTÈME D'ANALYSE D'IMPACTS DE CAVITATION
}

\author{
O. BASSET, F. DENIS et G. GIMENEZ \\ Laboratoire "Traitement du Signal et Ultrasons", INSA Bât. 502, 20 \\ Avenue Albert Einstein, F-69621 Villeurbanne Cedex, France
}

Résumé - Le système réalisé est destiné à analyser des séries d'impacts de cavitation dont les caractéristiques se modifient d'un impact à l'autre. Ces caractéristiques sont : la localisation de l'impact, l'instant auquel il se produit et sa violence. Le système utilise une matrice de capteurs sur laquelle se produisent les impacts. Ce capteur composé de 16 éléments est connecté à un dispositif électronique de traitement des signaux. Les données sont ensuite transférées sur un micro-ordinateur (compatible PC). Ce système permet deux modes de fonctionnement : l'analyse des caractéristiques des impacts et l'enregistrmeent des signaux délivrés par les capteurs.

Abstract - This work presents a device devoted to track the time evolution of the "intensity" and space location of cavitation impingements on a test area. This real time device provides, for each event : the occuring instant, the particular location on the test area, a quantitative indication on the violence of the impingement. Actually, the test area is an array of 16 transducers connected to a processing device which extracts the preceding parameters. The data are then transfered to a computer (PC compatible). This device includes two independant sections : an analysing section and a recording section.

\section{1 - INTRODUCTION}

Il existe peu d'instruments et de méthodes permettant d'étudier la cavitation. L'exposition d'un échantillon à la cavitation est le moyen le plus répandu. Après quelques heures d'exposition, on observe la surface de l'échantillon au microscope pour déterminer le nombre, la profondeur ou la forme des cratères. On détermine aussi la masse perdue [1]. Cependant, au cours de l'expérience les impacts ont pu être plus ou moins agressifs, la zone de cavitation a pu se déplacer. Tout cela n'est pas accessible car l'observation ou la mesure de la perte de masse intègre ces effets.

D'autres méthodes donnent des résultats instantanés. Elles utilisent différents capteurs : par exemple l'effet électrique lié aux impulsions [2] ou l'analyse de l'amplitude des signaux fournis par des sondes piézoélectriques en réponse à la cavitation [3, 4].

Ce travail présente un système destiné à suivre dans le temps l'évolution de l'intensité et de la localistion d'impacts de cavitation sur une surface-test. Ce système temps-réel fournit pour chaque évènement son instant d'occurence, sa localisation particulière sur la surface et donne une indication quantitative de la violence de l'impact.

En réalité, la surface-test est la face sensible d'un transducteur matriciel. Ce capteur est connecté à un système de traitements qui extrait les paramètres intéressants. Un premier dispositif fonctionnant avec un capteur matriciel de 9 éléments a été réalisé. Ce dispositf a été testé en milieu cavitant dans une veine-tourbillon. Les résultats encourageants nous ont amené à développer un dispositif plus performant fonctionnant avec un capteur matriciel de 16 éléments. En plus de la fonction "analyse temps réel", ce système propose la numérisation simultanée des 16 voies. 


\section{2 - PRINCIPE}

Le dispositif mis en oeuvre se compose de trois parties : un capteur matriciel, un système électronique et un micro-ordinateur (cf Fig. 1).

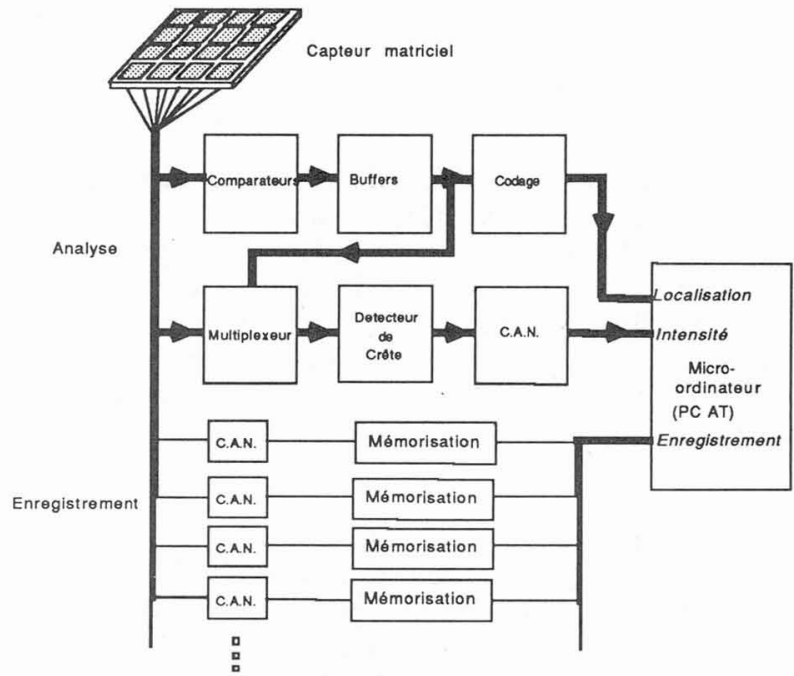

Figure 1 : Schéma synoptique de fonctionnement du système

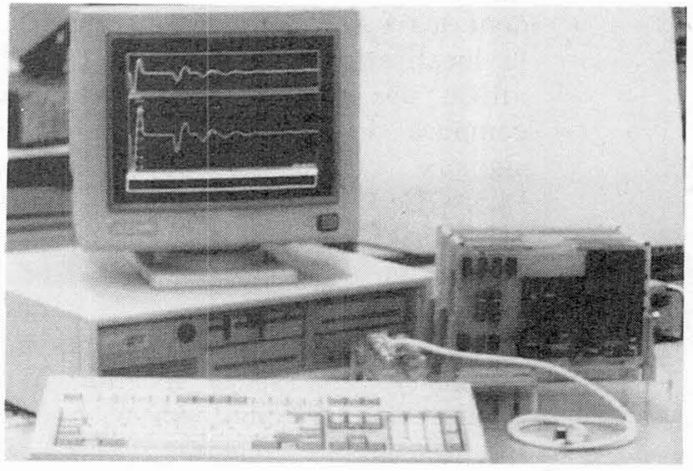

Figure 2 : Système d'analyse d'impacts

La surface soumise aux impacts est recouverte de petits transducteurs (éléments piézoélectriques carrés) jointifs mais indépendants formant un réseau bi-dimensionnel. Les éléments de ce capteur sont connectés à un système électronique qui permet deux modes d'utilisation : l'analyse des impacts et l'enregistrement des signaux. La fonction analyse d'impacts fournit en temps réel, c'est à dire après chaque évènement, des informations sur la localisation et l'intensité du choc. Ce système localise les impacts en identifiant le capteur le plus proche du choc. C'est en effet le signal délivré par ce capteur qui franchit le premier un seuil de tension pré-défini. La localisation de la source est donnée par la position de ce capteur. La résolution et la précision de ce système sont ainsi liées à la densité de capteurs implantés. L'incertitude sur la localisation est égale à la taille d'un capteur élémentaire. Le signal produit sur le capteur identifié est sélectionné par un multiplexeur analogique et envoyé sur un détecteur de crête afin de quantifier la violence du choc. Celle-ci est estimée à partir de la plus forte amplitude atteinte par le signal. Cette valeur de tension, fournie par le détecteur de crête, est convertie en une valeur numérique qui permet de définir la classe d'intensité du choc.

A ce moment les informations extraites par le système électronique sont codées sur des mots binaires. Le micro-ordinateur va alors lire ces informations avant de les traiter, pour une utilisation temps réel, ou de les stocker en vue d'un traitement ultérieur. L'instant d'apparition de l'évènement peut également être enregistré.

$\mathrm{La}$ fonction enregistrement des signaux permet de numériser et de stocker les $\mathrm{N}$ signaux issus des $\mathrm{N}$ éléments du capteur. Cela permet de connaître, à chaque instant, la répartition des pressions agissant sur la surface test. Les signaux sont numérisés sur 16384 points avec une résolution de 8 bits puis sont intégralement transférés sur ordinateur. Ils sont alors disponibles pour toutes sortes de traitement, en temps différé cette fois (par exemple localisation du premier capteur atteint, analyse d'amplitude...). 


\section{3 - TEST EN VEINE TOURBILLON D'UN ANALYSEUR NEUF VOIES}

Un premier système a été réalisé permettant de tester la fonction analyse d'impacts. Le capteur associé à ce système est une matrice carrée de 9 éléments $(3 \times 3)$ de $3 \mathrm{~mm}$ de coté. La surface contrôlée est donc environ $1 \mathrm{~cm}^{2}$. Ce système permet de classer les chocs selon trois catégories : les forts, les moyens et les faibles. Il a été testé au moyen d'un dispositif cavitant : la veine tourbillon. C'est un système générateur d'impacts de cavitation individualisés dont la fréquence et la violence sont réglables [5]. Tous les impacts se produisent sur une surface circulaire de $8 \mathrm{~mm}^{2}$ environ.

L'observation des signaux délivrés par les capteurs, en réponse aux impacts de cavitation, montre qu'un impact est suivi d'un certain nombre de rebonds d'amplitude maximale décroissante. Le temps de traitement des informations (acquisition et mémorisation) est suffisamment court (inférieur à $1 \mathrm{~ms}$ ) pour que tous les rebonds soient pris en compte. Notons que l'utilisateur peut choisir de ne tenir compte que des premiers impacts de chaque évènement (en inhibant le système un certain temps après chaque acquisition).

La figure 3 montre la répartition moyenne des impacts détectés lors d'un essai. On peut remarquer qu'existent des zones d'impactage préferentielles, $60 \%$ des chocs sont localisés sur le capteur $\mathrm{n}^{\circ} 4$. Les éléments des angles de la matrice sont peu atteints. Cette répartition est conforme à l'observation des échantillons métalliques érodés dans la veine.

\begin{tabular}{|r|r|r|}
\hline 7 & 4 & 1 \\
$0.3 \%$ & $60 \%$ & $6 \%$ \\
\hline 8 & 5 & 2 \\
$3 \%$ & $12 \%$ & $6 \%$ \\
\hline 9 & 6 & 3 \\
$0.3 \%$ & $12 \%$ & $0.3 \%$ \\
\hline
\end{tabular}

Figure 3 : Répartition moyenne des chocs

La modification de l'intensité des impacts, réalisée par variation de la fréquence n'a pas permis d'observer une évolution dans la distribution spatiale des chocs.

Le système de localisation offre une très bonne discrimination du capteur atteint. En effet, il arrive très rarement qu'un choc soít localisé sur deux capteurs contigus. (moins de $1 \%$ des cas).

Les réponses des capteurs aux chocs présentent une amplitude très élevée (parfois plus de $12 \mathrm{~V}$ d'amplitude maximale). Afin que l'amplitude maximale ne dépasse pas les capacités des convertisseurs, une mise en forme des signaux est nécessaire. Le classement des impacts par intensité a permis de constater que dans la plupart des cas, pour un évènement de cavitation, le premier collapse détecté est moins violent que le second. Les rebonds suivants ont une amplitude décroissante. La configuration de la veine permet d'expliquer ce phénomène [6].

\section{4 - DEVELOPPEMENT D'UN SYSTEME D'ANALYSE 16 VOIES}

Les résultats intéressants obtenus lors des essais de faisabilité nous ont encouragé à développer un système plus performant.

Le capteur matriciel comporte 16 éléments $(4 \times 4)$ de $2 \mathrm{~mm}$ de côté. Le système électronique propose deux modes de fonctionnement : analyseur d'impacts et enregistreur 16 voies. L'analyse d'impacts donne la localisation des chocs et indique le niveau d'amplitude maximale atteint parmi 14 classes. La fonction enregistrement permettra de numériser et de 
stocker les 16 signaux issus des 16 capteurs. Actuellement, une voie seulement est disponible.

Quelques paramètres doivent être définis par l'utilisateur. La fréquence d'échantillonnage des signaux peut varier de 2 à $16 \mathrm{MHz}$, A la fréquence maximale, les signaux sont enregistrés durant $1 \mathrm{~ms}$. Bien sûr, cette durée augmente si la fréquence choisie est plus faible. Rappelons que les signaux des capteurs peuvent avoir des amplitudes très importante. Aussi, le système propose différentes atténuations ou amplifications du signal (de 0,1 à 2) qui permettent d'utiliser au mieux la dynamique des convertisseurs analogiquenumérique.

Les signaux sont enregistrés en utilisant une fonction "pré-déclenchement". Cette fonction permet d'enregistrer une partie des signaux précédant l'instant de déclenchement (c'est à dire l'instant ou l'amplitude d'un signal franchit un seuil de tension prédéfini). Ainsi, le début des signaux n'est pas perdu. Le système permet de choisir la portion de mémoire qui sera consacrée au signal avant déclanchement. Elle peut varier de $1 / 1024$ à $1 / 4$ de la profondeur mémoire totale $(16 \mathrm{k}$ octets). Les différents paramètres cités sont définis directement à partir de commandes informatiques.

Le micro-ordinateur utilisé pour le pilotage de ce système (analyseur et enregistreur) est un IBM PC équipé d'une carte d'interface Nautil LOGIC-40. Différents programmes ont été réalisés pour la présentation des informations. Par exemple, dans le cas de l'analyse d'impacts, on visualise 16 histogrammes, associés à chaque capteur sur lesquels on représente le nombre d'impacts localisés sur ce capteur en fonction de leur intensité.

\section{5 - CONCLUSION}

Un premier système d'analyse d'impacts de cavitation a été réalisé et testé en milieu cavitant. Il a démontré la faisabilité et l'intérêt d'un tel dispositif. Une version étendue a alors été développée, utilisant un capteur matriciel de 16 éléments. Des essais préliminaires ont permis de tester le bon fonctionnement du dispositif. D'autres essais vont être réalisés prochainement en milieu cavitant (veine tourbillon).

Indiquons que la forme et la taille des 16 capteurs peuvent être définis en fonction de la surface ou de la zone à surveiller. Par exemple, une barette linéaire de 16 éléments peut être intéressante pour certaines applications. 16 capteurs indépendants peuvent également être utilisés.

\section{REFERENCES}

1 - R.T. KNAPP, J.W. DAILY, F.G. HAMMITT, In "Cavitation", Mc Graw-Hill, 1970.

2 - L. CHINCHOLLE, "Use of a novel apparatus measuring the instantaneous erosion rate by an abrasion (sand) a by cavitation", The Symposium Hydraulic for hight dams, Pekin, Nov. 88.

3 - V.K. MAKAROV, A. A. KORTNEV, A. G. SUPRUN, G.I. OKOLELOV, Proc. 6th ISNA Symp., Moscou USSR, vol.2, pp. 104-115, 1975.

4 - G. GIMENEZ, J. MARCOZ, "Amplitude distribution of pulses produced by schok waves due to cavitation bubbles", J. Acoust. Soc. Am. 67 (4), April 1980.

5 - Y. LECOFFRE, J. MARCOZ, B. VALIBOUSE, "Generator of Cavitation Vortex", ASME Symposium Cavitation Erosion in Fluid Systems, Boulder, Colorado, 1981.

6 - J. L. HEUZE, "Endommagement par erosion de cavitation de métaux et alliages de structure cubique à faces centrées", Thèse de l'Université Paris VI, 1988. 